\title{
MULTIPLE-POINT GEOSTATISTICS TO DERIVE MISSING SURFACE DISPLACEMENT VALUES OF A GLACIER INFERRED FROM DINSAR
}

\author{
B. Ranjit ${ }^{1, *}$, V. A. Tolpekin ${ }^{2}$, A. Stein ${ }^{2}$ \\ ${ }^{1}$ Land Management Training Centre, Ministry of Land Management, Cooperatives and Poverty Alleviation, Dhulikhel, \\ Kavrepalanchok, Nepal - ranjit.bhuwan@gmail.com \\ ${ }^{2}$ Dept. of Earth Observation Science, Faculty of Geo-Information Science and Earth Observation (ITC), University of Twente, \\ Hengelostraat 99, 7514 AE Enschede, The Netherlands - (v.a.tolpekin, a.stein)@utwente.nl
}

\section{SarCon}

KEY WORDS: Glacier displacements, DInSAR, Kriging, Multiple-point geostatistics, Direct sampling

\begin{abstract}
:
Glacier displacements play a vital role in the monitoring and understanding of glacier dynamics. Glacier displacement fields are typically retrieved from pre- and post-event SAR images using DInSAR. The glacier displacement map produced by DInSAR contains missing values due to decorrelation of the SAR images. This study demonstrates the utility of direct sampling-a wellestablished multiple-point geostatistics method-for deriving those missing values. Univariate and bivariate implementations of direct sampling are employed. In the univariate implementation, missing values are derived in single displacement map, whereas in bivariate implementation gaps in two displacement maps are filled simultaneously. Evaluation is carried out by artificially generated missing values on the displacement map of different shapes and sizes at different locations with known values. Imposed missing values are then reconstructed and compared with the original values. Reconstruction results of both implementations were compared with ordinary kriging using qualitative and quantitative measures. The study shows that with an increase in the size of such discontinuities, ordinary kriging predictions deteriorate significantly, whereas only slight decrease in reconstruction accuracy is observed for direct sampling. The results of both implementations are similar with the univariate implementation performing slightly better over bivariate implementation because the information from ancillary data is only partly complementary for bivariate reconstructions. Direct sampling performed better than ordinary kriging with accuracy below the DInSAR detection limit. This study concludes that multiple-point geostatistics is an effective method for deriving missing values in DInSAR derived displacement maps. Direct sampling based reconstruction is fast and straightforward to implement.
\end{abstract}

\section{INTRODUCTION}

Glaciers are masses of ice formed by the accumulation and compaction of snow over a long duration of time. They constantly move because of stresses induced by their weight and gravity. Information on glacier velocity is vital for studying the glacier dynamics, glacier mass balance models, monitoring the glacier response to climate change, understanding the internal stresses and strains caused by gravity-induced flow, glacier hazard prediction and gathering insight on seasonal variability of glacier (Joughin et al., 2010).

Satellite remote sensing techniques-feature tracking in both optical and Synthetic Aperture Radar (SAR) images, and interferometric SAR (InSAR)—-have been applied for deriving surface velocity of mountain glaciers over in situ measurements because in situ measurements are costly, time-consuming, limited over a small geographical area, and impractical to perform in inaccessible, remote and vast mountain glaciers (Joughin et al., 2010). Feature tracking in optical images, especially in the mountainous region, is problematic due to cloud cover. Feature tracking in SAR images and InSAR techniques eliminate this limitation as SAR images are independent of sun-illumination, penetrate clouds and can function day and night in all-weather condition. However, feature tracking on SAR images rely on detectable surface features in the images, thus, fails in the area without distinct surface features like crevasses and its detection limit depends on the pixel size of the SAR images. InSAR does not suffer from these drawbacks. Above all, glacier displacement estimates from the InSAR is considerably more accurate and precise-in the order of few centimetres - compared to feature tracking. Thus, InSAR technique is greatly valued for glacier velocity studies.

InSAR uses the phase information of the radar images of the same scene acquired from two positions using two receiving antennas separated either in time (repeat pass acquisition) or space (along-track or across-track acquisition). Most of the SAR sensors provide images of same scenes acquired at some time apart so repeat pass interferometry has been extensively applied for glacier surface displacement estimation (Schneevoigt et al., 2012). Amongst current satellite sensors, Sentinel mission is the latest and the most promising source for repeat pass SAR data. Thus, employing differential interferometric SAR (DInSAR), glacier surface displacement is derived exploiting enhanced interferometric capability of freely available Sentinel-1 (S1) SAR image pair with the shortest repeat pass of 6 days in this study.

The two SAR images must be coherent to form interferogram which comprises phase difference information. Loss of coherence with time is caused by snow and ice melting, wind induced snow drift, precipitation in form of snow or rain and gradient of displacement greater than half a fringe per pixel. Temporal decorrelation produces unreliable interferometric results. Thus, the area with low value of coherence are avoided and masked out during phase unwrapping process (Schneevoigt

\footnotetext{
* Corresponding author
} 
et al., 2012). As a consequence, the glacier surface displacement map derived from DInSAR consists of missing values (gaps) in incoherent areas. Reconstruction of these spatial discontinuities is of high importance in glacial studies to integrate these data with modelling frameworks requiring continuous data fields and to assess the spatial and temporal patterns of the glacier displacement (Mariethoz et al., 2012).

Deterministic and geostatistical interpolation methods are available for gap-filling. Deterministic techniques use a mathematical function to interpolate the values at unsampled location, based on either the degree of similarity as in Inverse Weighted Distance (IDW) or the degree of smoothing as in Radial Basis Function (RBF) in relation with neighbouring data points. However, these deterministic methods are unable to provide uncertainty estimates. Whereas, geostatistical methods are based on statistical models performing stochastic predictions of values at unknown locations, and therefore can provide spatial model of uncertainty. However, traditional geostatistics is based on Random Function (RF) model parameterized by semi-variogram and covariance. Therefore, spatial variability is captured by only considering two spatial locations at one time. This results in consideration of only linear relationship with covariates. So, the spatial dependency of phenomenon exhibiting a much stronger correlation at higher order cannot be described by two-point statistics (Mariethoz et al., 2010). Recently developed Multiple-point geostatistics (MPS) method, belonging to family of non-parametric geostatistical methods, can solve this problem because spatial variability is modelled using training images (TIs), from which spatial structures and patterns are borrowed. Thus, this study investigates the possibility of reconstruction of the gaps caused by loss of coherence in DInSAR derived displacement maps of glaciers generated from S1 image pair and predict the missing data using Direst Sampling (DS) MPS method.

\section{STUDY AREA AND MATERIALS}

\subsection{Study Area}

Apart from Polar region, Himalaya is one of the widely glaciated areas in the globe. In recent years, these mountain glaciers are becoming vulnerable to climate change. It is getting important to monitor these glaciers' displacements for anticipating possible future catastrophe, early warning of potential glacial lake outburst flood, mass balance studies and understanding glacier dynamics. Thus, Ngozumpa glacier, which lies in Dudh Koshi basin of Himalaya at $28^{\circ} 00^{\prime} \mathrm{N}$ longitude and $86^{\circ} 45^{\prime} \mathrm{E}$ latitude is chosen as the study area. Figure 1 shows the study site in band combination used for glacier and snow mapping in the Sentinel-2 optical image (bands 11,8A, 3 as RGB), where snow is seen in light blue, glacier in dark blue and debris-covered area and surrounding moraine in red.

\subsection{Data Description}

The details of the SAR image pairs fulfilling the pre-requisites for DInSAR chosen for the study are in Table 1. For obtaining the coherent image pair, SAR images with the least temporal baseline of 6 days of $\mathrm{S} 1$ and of the coldest time of the year but with no precipitation were selected. Additionally, the image pairs were checked to be acquired by the identical satellite in the same nominal orbit using same acquisition mode and properties like beam, polarization, off-nadir angle, etc. with shorter baseline than that of the critical baseline. The downloaded images were level-1 SLC product comprising geo- referenced focused SAR data with preserved phase information, hence, suitable for interferometric processing. Even though SAR images over AOI were available at dual polarization (VV and $\mathrm{VH}$ ), VV channel was used over cross-polarized $\mathrm{VH}$ as it yields the highest coherence due to higher SNR ratio and less back-scattering variation.

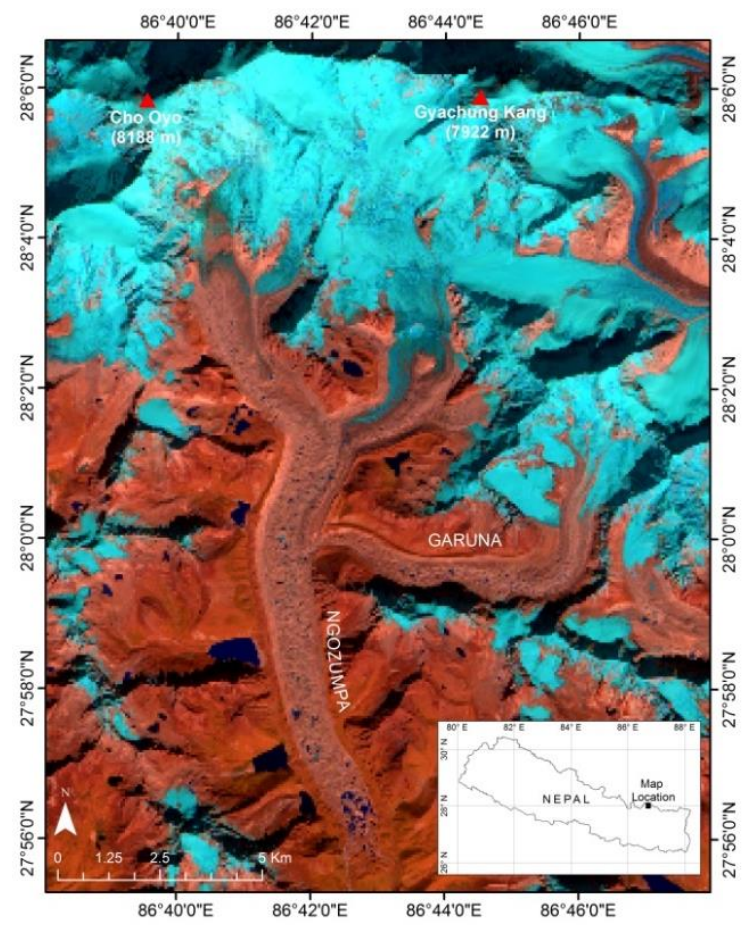

Figure 1. Location of Ngozumpa glacier, Nepal shown in Sentinel-2 false colour image (Level-1C bands 11, 8A, 3 as RGB) date 2016-10-30

\section{METHODOLOGY}

\subsection{DInSAR}

At first, the processing chain of DInSAR is adopted to derive surface displacement maps from the SLC SAR focused image pairs I and II using Sentinel Application Platform (SNAP) version 4.0.0. Explaining DInSAR in-depth is beyond the scope of this paper and the readers are referred to Schneevoigt et al., (2012). DInSAR method of obtaining line of sight (LOS) displacement maps is included in brief summary here. First, the image pair is co-registered by using an earlier image as the master to align the pixels of the latter slave image at sub-pixel accuracy to obtain positive deformation in time. From the coregistered image pair, an interferogram is formed by the multiplication of the master image with the complex conjugate of the slave image. In the interferogram, the contribution in the interferometric phase by the topography, flat earth phase and other sources of noise are removed by means of SRTM 3-sec DEM, the precise orbital and metadata information, and Goldstein Filter respectively. The meteorological condition of the study area is assumed to be stable. Thus, atmospheric phase delay is negligible. After the removal of phase of these contributors, the interferometric phase due to LOS surface displacement is obtained. The statistical-cost, network flow algorithm SNAPHU was used for phase unwrapping process of the interferogram (Chen \& Zebker, 2002). As low coherence value cannot be unwrapped, Schneevoigt et al. (2012) suggested masking them out during phase unwrapping. The phases having coherence lower than the threshold $(<0.25)$ were not considered for phase unwrapping. The displacement value obtained so far is 


\begin{tabular}{llllllll}
\hline $\begin{array}{l}\text { SAR Image } \\
\text { Pairs }\end{array}$ & Master image & Slave image & $\begin{array}{l}\text { Perpendicular } \\
\text { Baseline }(\mathbf{m})\end{array}$ & Track & $\begin{array}{l}\text { Temporal } \\
\text { Baseline } \\
\text { (days) }\end{array}$ & $\begin{array}{c}\text { Polarization } \\
\begin{array}{l}\text { Ascending/ } \\
\text { Descending } \\
\text { Orbit }\end{array}\end{array}$ \\
\hline Pair I & $2016-10-27$ (S1-A) & $2016-11-02(S 1-B)$ & +32.45 & 121 & 6 & VV & Descending \\
Pair II & $2016-11-02$ (S1-B) & $2016-11-08(S 1-A)$ & -42.13 & 121 & 6 & VV & Descending \\
\hline
\end{tabular}

Table 1. List of S1 SAR image pairs used for InSAR of the Ngozumpa glacier

relative. The phase difference at the reference point-rocks on the sides of the glacier-should always be zero. Absolute displacement map was obtained by deducting the value of the chosen reference marker. The unwrapped interferogram was geocoded using SRTM DEM and Range-Doppler approach such that the map coordinates were projected to UTM zone 45-north and WGS-84 datum. In this way, geocoded displacement map was obtained with gaps at incoherent areas filled using DS afterwards.

\subsection{Direct Sampling}

The basic principle of the DS method is to use TI to identify spatial features and properties to fill the gaps. The missing values of the LOS displacement map are sequentially replaced by matching the patterns of the TI with the values of the neighbouring pixels. Hereafter, the glacier displacement image with gaps to be reconstructed is addressed as the target image, while the image providing information for filling gaps in target image is referred to as the input image.

Let $Z(x)$ be the variable of interest to be simulated, where the gapped pixel in the target image is denoted by $x$. Similarly, $N_{x}$ is the ensemble of the $n$ closest pixels of $x$ that are informed. These $n$ pixels define the neighbourhood. The concept of DS method is to find one possible outcome of $Z$ conditional to $N_{x}$ from the conditional cumulative function given in Equation 1.

$$
F(z)=\operatorname{Prob}\left(Z(x) \leq z \mid N_{x}\right)
$$

The basic idea is to find another pixel $y$ in the TI (in this study case input image) that has neighbouring pixels $N_{y}$ similar to $N_{x}$. The distance $d\left(N_{x}, N_{y}\right)$ is used to compare the similarity between $N_{x}$ and $N_{y}$. The concept of distance is flexible and can be applied to both categorical and continuous variables. Since the variable of concern in this research is continuous, the distance adopted was the Weighted Euclidean distance, as suggested by Mariethoz et al. (2012) to be used for continuous variable. The Equation 2 is used to compute the distance.

$$
d\left(N_{x}, N_{y}\right)=\frac{1}{\eta} \sqrt{\sum_{i=1}^{n} w_{i}\left[Z\left(x_{i}\right)-Z\left(y_{i}\right)\right]^{2}}
$$

where $w$ is weight of each node and $\eta$ is normalization factor applied so that the value of distance is bounded in the interval $[0,1]$. It is the maximum difference between the two values of $Z$ in the TI.

The path followed in search for $y$ in the TI can be random or unilateral. In this study, random search path has been used. In case of continuous variable, the perfect match between the data events in the TI and SG is often not found which is why an acceptable threshold $t$ is introduced. During the scanning process of TI, when the pixel $y$ is found in the TI with the distance smaller than predefined threshold $t$, the value $Z(y)$ is picked and assigned to $Z(x)$. If the search area has reached a predefined maximum search fraction $f$ of the TI but unable to find a pixel $y$ satisfying the threshold requirement, then the pixel $y$ with the lowest distance is accepted and its value $Z(y)$ is assigned to $Z(x)$. Figure 2 graphically illustrates the DS process. The data event is defined in Figure 2 (a) and the central pixel with a question mark represents the target pixel to be filled, and the black and the two white pixels are neighbourhood with known values from either previous simulation or are conditioning data assigned to SG prior to the simulation. Here, a categorical case where a pixel can take only two values-0 (white) and 1 (black) is dealt with. Figure 2 (b) shows how the search window is defined in the TI grid by using the dimensions a, b, c, d of the data events from Figure 2 (a). Figure 2 (c) shows carrying out search in the search window of TI using data events. The search moves to next location following random path until the simulation data event is matched satisfactorily as shown in Figure 2 (d). Then the value of the central pixel of the first matching data event is assigned to the target pixel Figure 2 (e). In this case, the data event in the TI and the data event in the SG match exactly hence the distance is zero and the value $\mathrm{Z}(\mathrm{y})=1$ is assigned to the SG. The DS method is easily extended to the multivariate case. The distance between multivariate neighbourhoods is computed by a weighted average of the distances taken individually for each univariate neighbourhood. Equation 3 gives the distance equation for multivariate case.

$$
d\left(N_{x}^{\prime}, N_{y}^{\prime}\right)=\sum_{j=1}^{m} \frac{\alpha_{j}}{\eta_{j}} \sqrt{\sum_{i=1}^{n} w_{i}^{k}\left[Z^{k}\left(x_{i}\right)-Z^{k}\left(y_{i}\right)\right]^{2}}
$$

where $m$ is the number of variable, $\alpha_{j}$ and $\eta_{j}$ are the weights and the normalization constant for each variable respectively.

\subsubsection{Training Image}

Since TIs should contain the variability, connectivity and structural properties of the phenomenon under investigation and DS can perform simulations using incomplete TIs, the LOS displacement maps generated from DInSAR with the no data values in masked out incoherent area were used as the TIs.

\subsubsection{DS Cases}

Two different DS cases were considered during the reconstruction process. Case 1: DS Univariate simulation $\left(\mathrm{DS}_{\mathrm{u}}\right)$ in which the non-gapped informed large portion of the displacement map itself was used as TI to reconstruct the gapped regions of the same displacement map. Case 2: DS Bivariate simulation $\left(\mathrm{DS}_{\mathrm{b}}\right)$ co-simulates to reconstruct the gaps in both displacement maps obtained from pair I and pair II together. For $\mathrm{DS}_{\mathrm{b}}$, equal importance to both gapped displacement maps were provided by supplying identical values of 0.5 for the weights associated with each displacement map.

\subsubsection{Ordinary Kriging for benchmarking}

Ordinary Kriging (OK) is the most commonly used kriging method. Moreover, in previous studies carried out by 


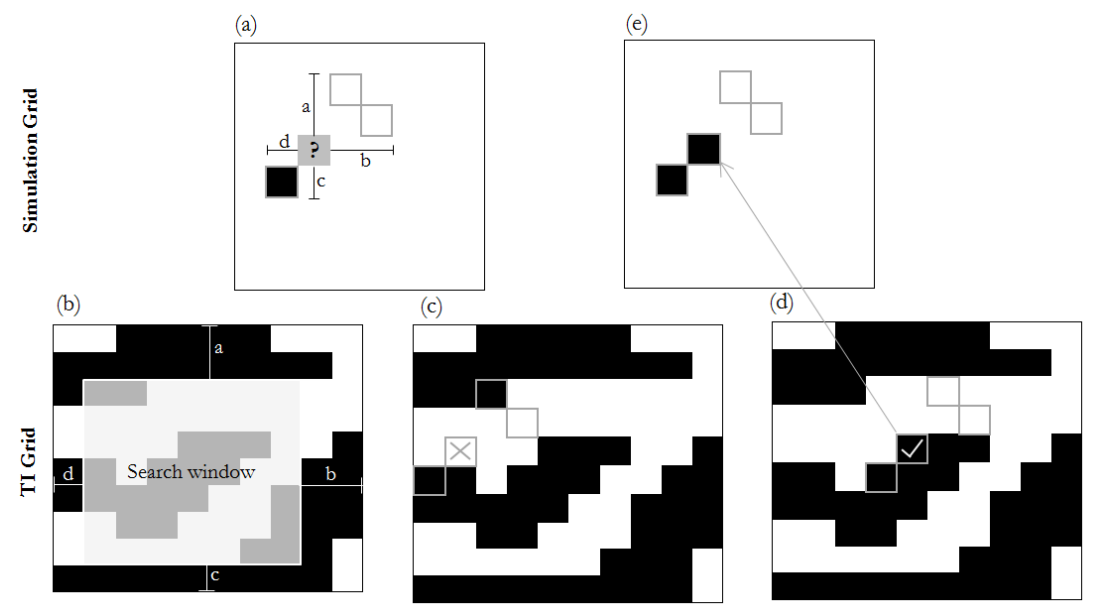

Figure 2. Graphical illustration of DS method. (a) Define the data event in the target image. (b) Define a search window in the TI grid. (c) Scan the TI using the search window until (d) the simulation data event is matched satisfactorily. (e) Assign the value of the central pixel of the first matching data event to the target pixel (Mariethoz et al., 2010)

Yaseen et al., (2013), the missing values in the InSAR derived LOS displacement map were interpolated using OK. For these reasons, OK has been used for benchmarking the results of DS. Since OK is a well-established method, its procedure is not explained here and readers can refer to literatures for in-depth explanation.

\section{RESULTS}

The interferograms of Ngozumpa glacier, before unwrapping, computed from SAR image pair I and II are shown in Figure $3(\mathrm{a})$ and 3(b) respectively. Figure 3(c) and 3(d) illustrate the coherence images of the SAR image pair I and II. It is observed that the coherence of the interferogram is high in the terminus and middle region of the glacier which gradually decreases further towards the upper region. Similarly, the noise in the interferograms corresponds with snow covered upper area of the glacier with low coherence. The interferograms shown in Figure 3(a) and 3(b), after being unwrapped and geocoded, produced the glacier displacement maps as seen in Figure 3(e) and 3(f). The location of the missing values in the displacement maps (at coherence $<0.25$ ) from pair I and II can be seen in the displacement maps. Due to high coherence, the gaps in the displacement at the debris-covered tongue of the glacier are few and small in size. Large gaps are present at low coherent upper region on the glacier covered by snow/ice (blue coloured part of the glacier in Figure 1).

For DS based reconstruction, optimal parameters were found by experimentation and then, used to generate 10 stochastic simulations. The average of the 10 simulated values corresponding to each missing value was taken as final result for both cases. To perform OK based gap-filling, for each gapped displacement maps from pair I and II, separate variogram analysis was performed to determine the fitting theoretical model. Afterwards, the missing displacement values in both displacement maps from pair I and II were interpolated using the respective modelled variogram. To qualitatively and quantitatively evaluate the performance of OK and DS, artificial gaps were created at locations with known displacement values. Imposed gaps were filled then validated against the known displacement values prior to the imposition of the artificial gaps. From a quantitative perspective, RMSE was employed as it is widely used performance validation measure in similar simulation studies. However, RMSE is sensitive to occasional large error. Additional measures namely histogram of the residuals and scatterplots were applied to evaluate the reconstruction results. To qualitatively assess the reconstruction results, visual inspection of existence of artifacts in resulting gap-filled displacement maps and residual distribution maps were carried out. Here, only selected measures are presented.

Small gaps can be repaired easily with high quality because the surrounding data provide sufficient information. So, bigger gaps occurring in the displacement maps with different shapes and sizes were picked and shifted to non-gapped area to create artificial gaps. Firstly, the mean gap size was picked for the analysis because most of the occurring gaps were of or around this size and also the gaps should not be small as mentioned earlier. The mean gap size was imposed at 12 different locations as seen in Figure 3(e) and 3(f), hereon referred as 12 shifted polygons. Secondly, to assess the impact of the big gap sizes, additional two large polygons were chosen and artificial gaps were created by repositioning them at the key location. The key location is the active westerly tributary of Ngozumpa glacier, with comparatively heterogeneous displacement values shown in red box in Figure 3(f).

For the 12 shifted polygons, the RMSE of the three cases- OK Prediction, $\mathrm{DS}_{\mathrm{u}}$ and $\mathrm{DS}_{\mathrm{b}}$ are given in Table 2. The RMSE of both DS cases are much smaller than that of OK. Between DS $_{u}$ and $\mathrm{DS}_{\mathrm{b}}$, the RMSE of $\mathrm{DS}_{\mathrm{u}}$ is lower, with slight improvement. The histograms of residuals for pair II are presented in Figure 5. All histograms clearly show steeper and symmetrical distribution - with the two DS cases appearing narrower and more normally distributed. For all three cases, most of errors are concentrated in and around 0 and are mostly unbiased. For OK, the $(95 \%)$ most of the errors in the displacement values are within the range of $[-0.015 \mathrm{~m},+0.015 \mathrm{~m}]$, while the corresponding range for $\mathrm{DS}_{\mathrm{u}}$ and $\mathrm{DS}_{\mathrm{b}}$ is $[-0.005 \mathrm{~m},+0.005 \mathrm{~m}]$, with $\mathrm{DS}_{\mathrm{u}}$ having the steepest distribution than $\mathrm{OK}$ prediction and $\mathrm{DS}_{\mathrm{b}}$ simulation. From all quantitative measures, both DS cases demonstrated better prediction compared to OK. Among the two DS cases, $\mathrm{DS}_{\mathrm{u}}$ simulation showed slight improvement in reconstruction accuracy compared to $\mathrm{DS}_{\mathrm{b}}$ simulations for both pairs. Similar results were observed for pair I.

Since $\mathrm{DS}_{\mathrm{u}}$ performed better than $\mathrm{DS}_{\mathrm{b}}$, to assess the reconstruction result of growing gap size to OK only $\mathrm{DS}_{\mathrm{u}}$ was used. Table 3 shows the RMSE of $\mathrm{DS}_{\mathrm{u}}$ is significantly smaller than that of OK for all three shifted polygons. With the increase 


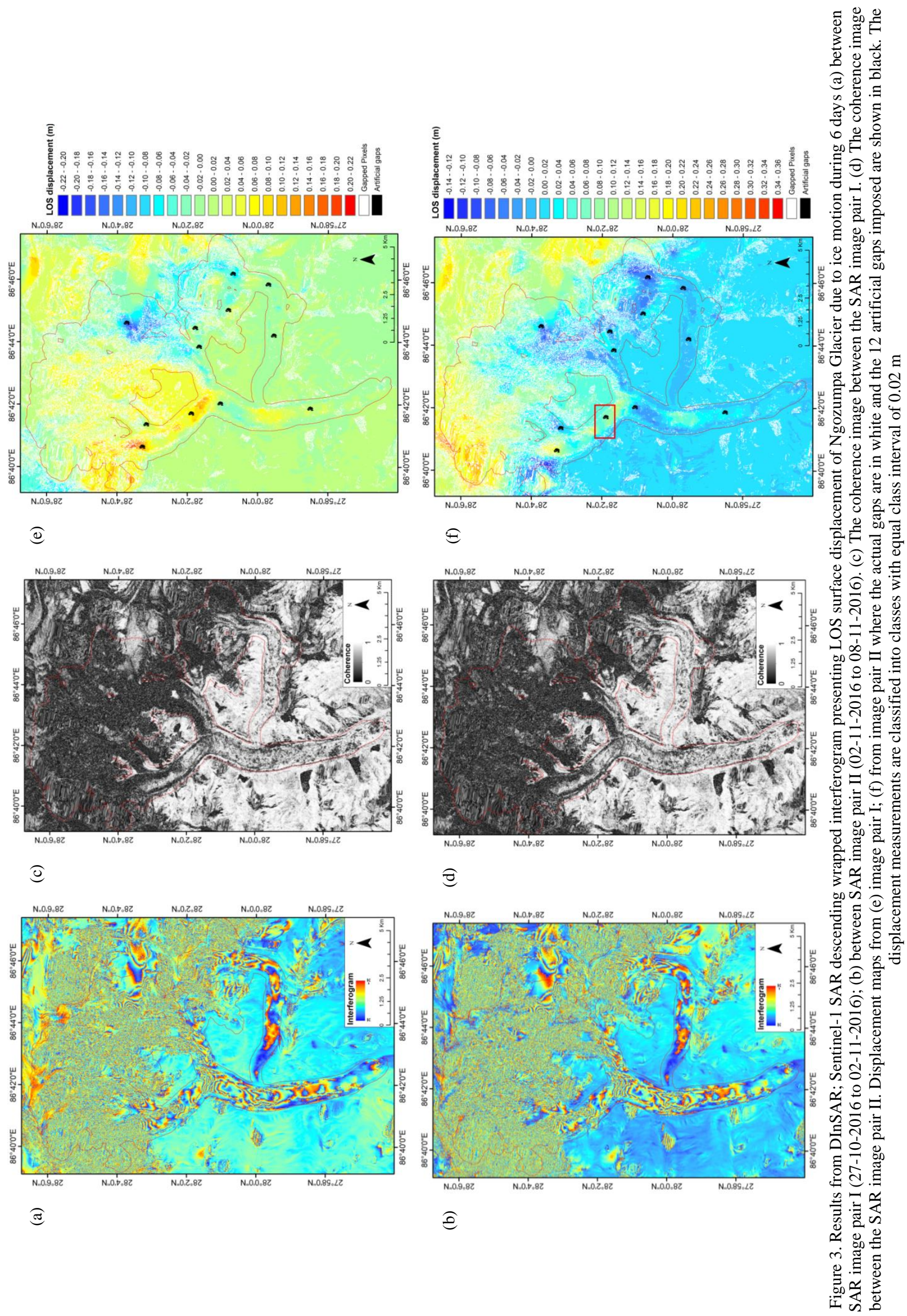



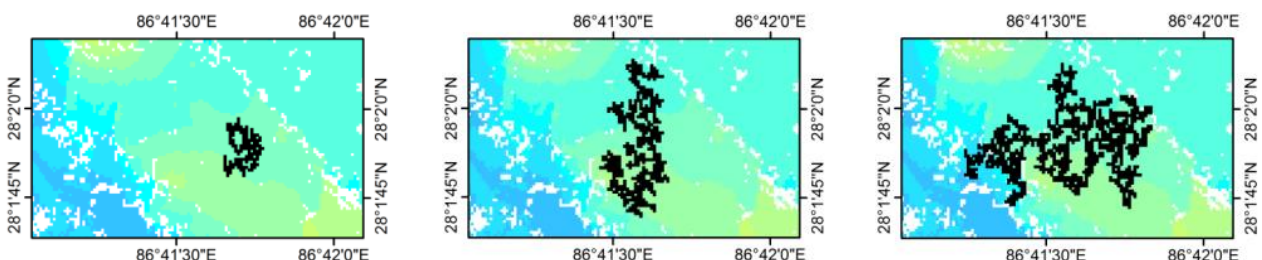

Figure 4. The polygons of increasing size imposed in key location of displacement map from pair II (shown in red box in Figure 3(f)) for accuracy assessment of growing gap size. Artificial gaps imposed are: polygon 1 (142 pixels), Polygon 2 (499 pixels) and Polygon 3 (1016 pixels) from left to right respectively shown in black

\begin{tabular}{llll}
\hline & \multicolumn{3}{c}{ RMSE } \\
\cline { 2 - 4 } & OK Prediction & DS Univariate & DS Bivariate \\
\hline Pair I & 0.00651 & 0.00213 & 0.00316 \\
Pair II & 0.00495 & 0.00164 & 0.00241
\end{tabular}

Table 2. Validation results-RMSE of OK prediction, $\mathrm{DS}_{\mathrm{u}}$ and $\mathrm{DS}_{\mathrm{b}}$ cases

in the gap size the accuracy degrades for both $\mathrm{OK}$ and $\mathrm{DS}_{\mathrm{u}}$. Yet, with growing gap sizes, the drop in RMSE for OK is sudden; while for $\mathrm{DS}_{\mathrm{u}}$ is small and gradual. The difference in RMSE (OK minus $\mathrm{DS}_{\mathrm{u}}$ ) increases with increasing gap size. This suggests OK performance for small gaps are satisfactory but with increasing gap size $\mathrm{OK}$ is unable to perform accurate prediction. On the contrary, $\mathrm{DS}_{\mathrm{u}}$ performs accurate prediction even with increasing gap sizes.

\begin{tabular}{llll}
\hline & \multicolumn{2}{c}{ RMSE } & $\begin{array}{l}\text { RMSE } \\
\text { difference } \\
\left(\mathrm{OK}-\mathrm{DS}_{\mathrm{u}}\right)\end{array}$ \\
\cline { 2 - 4 } & OK Prediction & DS Univariate & 0.00306 \\
Polygon 1 & 0.00369 & 0.00063 & 0.00704 \\
Polygon 2 & 0.00845 & 0.00141 & 0.00613 \\
Polygon 3 & 0.00834 & 0.00221 & \multicolumn{2}{c}{. }
\end{tabular}

Table 3. Validation results - RMSE of OK prediction and $\mathrm{DS}_{\mathrm{u}}$ cases

The histogram of residuals of $\mathrm{OK}$ and $\mathrm{DS}_{\mathrm{u}}$ for all three shifted polygons shown in Figure 6 clearly shows steeper, symmetrical and narrower distribution for $\mathrm{DS}_{\mathrm{u}}$ than $\mathrm{OK}$. In case of $\mathrm{OK}$, the range of the errors in the displacement values from polygon 1 to polygon 3 increased notably. In contrast, most of the errors in the displacement values are within range of $[-0.005,+0.005]$ for $\mathrm{DS}_{\mathrm{u}}$. All the accuracy assessment indicators suggested that the performance of $\mathrm{DS}_{\mathrm{u}}$ is superior to OK with growing gap sizes.
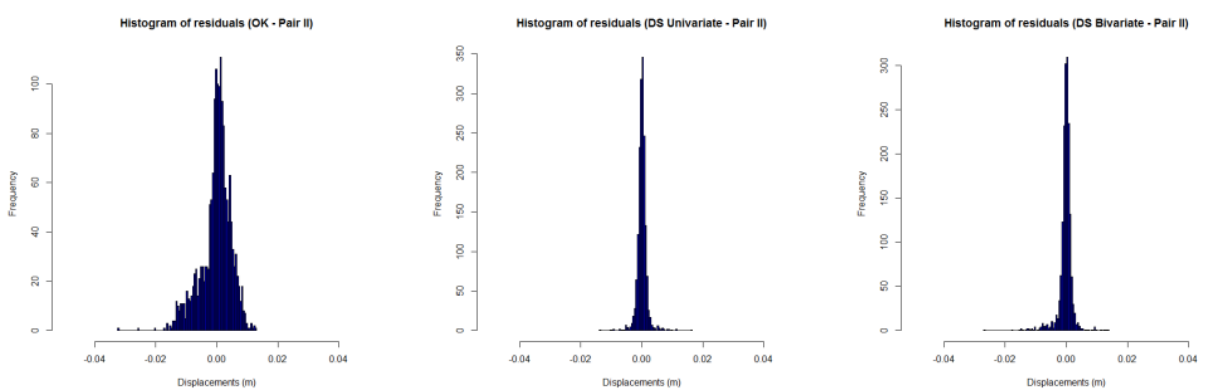

Figure 5. Histograms of residuals of all three cases (from left to right) $-\mathrm{OK}, \mathrm{DS}_{\mathrm{u}}$ and $\mathrm{DS}_{\mathrm{b}}$ - for displacements from pair II
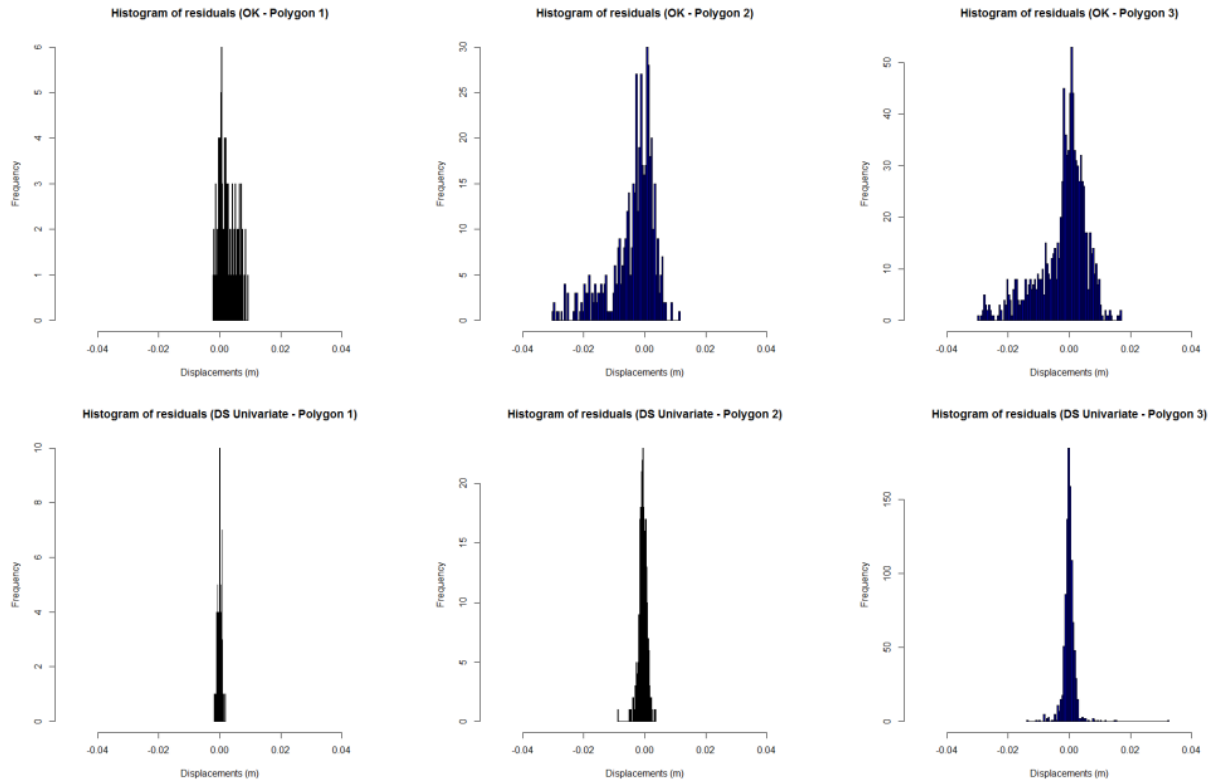

Figure 6. Histograms of residuals of $\mathrm{OK}_{\text {and }} \mathrm{DS}_{\mathrm{u}}$ for displacements from pair II. The three columns represent graphs for polygon 1 to polygon 3 (from left to right). 


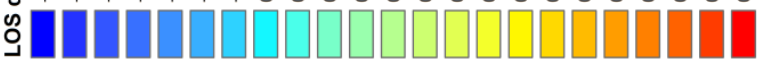
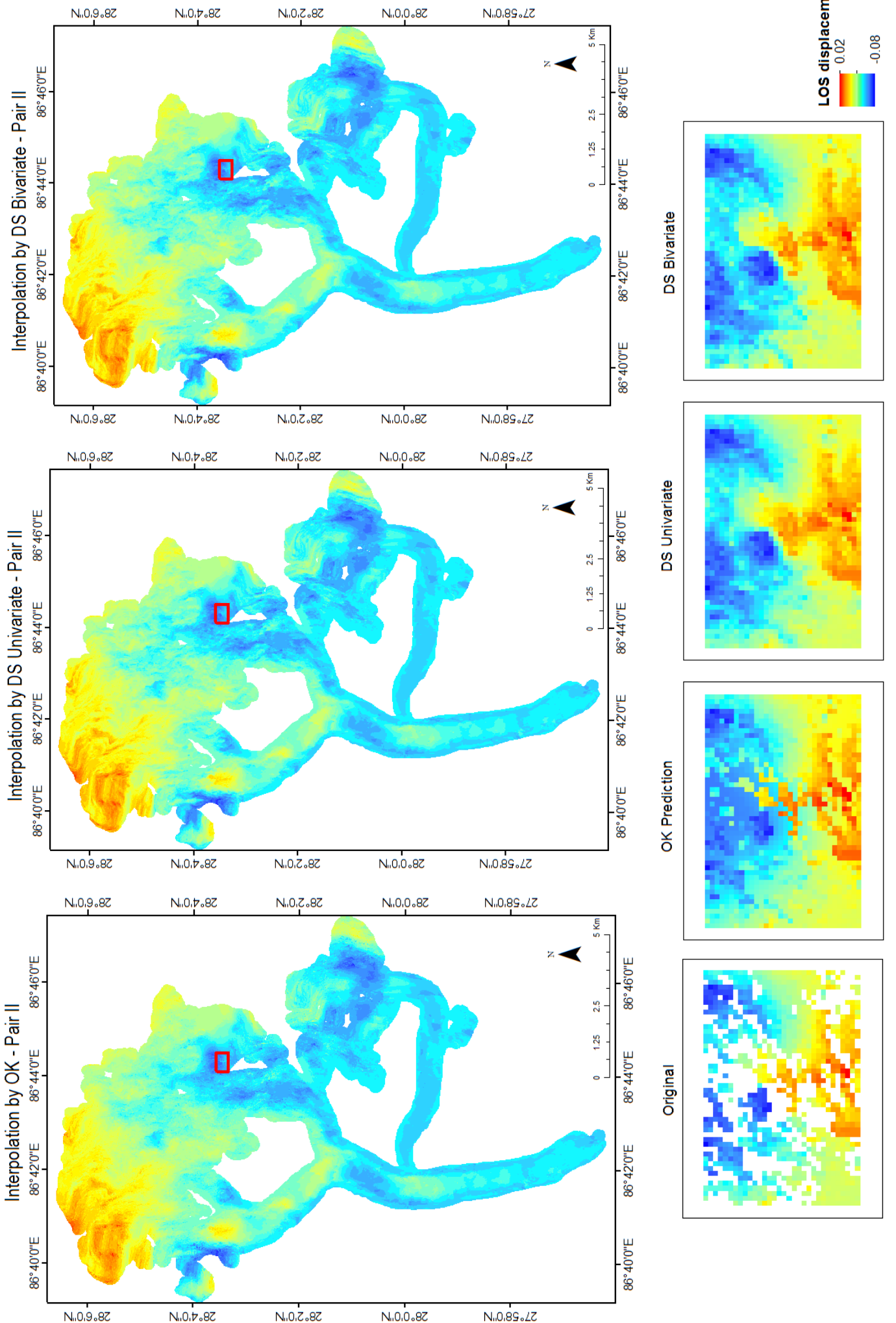

0ี 
By combining the predicted results of the missing displacement values with the known values of the glacial displacement map for all three cases- $\mathrm{OK}, \mathrm{DS}_{\mathrm{u}}$ and $\mathrm{DS}_{\mathrm{b}}$ - complete glacial displacement maps were produced. The prepared displacement maps from pair II are shown in Figure 7. To better compare the quality of gap filling of all three cases, a small portion of the filled displacement map from pair II was enlarged and presented at the bottom of Figure 7. Some noises were seen in the OK interpolated displacement map whereas the results from DS were relatively smooth. DS maps showed better preservation of the glacier displacement patterns than $\mathrm{OK} . \mathrm{DS}_{\mathrm{u}}$ and $\mathrm{DS}_{\mathrm{b}}$ filled displacement maps are both similar, with some subtle differences. This visual qualitative assessment concurs with the error statistics, with DS providing better gap filling results.

\section{DISCUSSION}

The quality of $\mathrm{OK}$, and of $\mathrm{DS}_{\mathrm{u}}$ and $\mathrm{DS}_{\mathrm{b}}$ reconstructions were assessed by validating against reference values for 12 shifted artificial polygons enforced at different locations. The two DS cases gave better results than $\mathrm{OK}$ for displacements from both image pairs. This can be attributed to the ability of DS to capture internal heterogeneity and multiple point dependencies of the glacier displacement field. The reconstruction of three selected polygons of increasing size shifted to a key location showed that the accuracy degrades for both DS and OK if gap size grows. The entire spatial structure may be missing in large gaps causing the reconstruction to be less accurate. Nevertheless, DS performed better than OK for large gaps, with only a slight drop in performance. The abrupt decrease in the performance of the $\mathrm{OK}$ is due to the increase in the degree of spatial heterogeneity in large gap sizes. Some structures present on either side of the small gap facilitate gap filling with realistic values (Mariethoz et al., 2012). Thus, OK gave good results for small gaps, whereas it cannot reproduce complex spatial patterns of large gaps. In contrast, MPS is able to resolve complex spatial patterns even in large gap sizes where OK fails. Thus, DS results are superior to OK with growing gap sizes.

MPS technique like DS compared to OK is straightforward to implement. In this study, OK was performed for entire displacement map at once, whereas OK performs better locally. For local interpolation, the displacement map should be divided into contiguous patches with sufficient sample points as in Yaseen et al., (2013). For each patch, an independent variogram analysis is to be performed. With numerous patches, this process is theoretically challenging as well as computationally costly. In contrast, MPS is simpler with its key concept of sampling spatial patterns from within TIs for predicting unknown values.

Most of the proposed gap-filling methods are limited to only one unknown variable to be reconstructed. Only DS has the capability to perform bivariate and multivariate simulations amongst the MPS methods till date. Further, conventional geostatistical methods like OK are not capable to perform gap filling in a bivariate and multivariate environment (Mariethoz et al., 2010). The potential of bivariate simulation of DS is demonstrated here by filling up gaps in both displacement maps simultaneously. The results of $\mathrm{DS}_{\mathrm{u}}$ and $\mathrm{DS}_{\mathrm{b}}$ were similar, with $\mathrm{DS}_{\mathrm{u}}$ providing slight improvements against $\mathrm{DS}_{\mathrm{b}}$. This may be attributed to the insufficient complementarity of the displacement characteristics of two maps (high temporal variability).

RMSE values showed that the accuracy of gap filling of DS were at the $\mathrm{mm}$ scale, whereas precision of DInSAR is at the $\mathrm{cm}$ scale. Thus, the obtained accuracy of gap filling by DS is acceptable and below the detection limit of DInSAR technique. For application of DS, fine-tuning three user defined parameters distance threshold $t$, search neighbourhood $n$ and scan fraction $f$ should be considered. Generally, small value of $t$ and large value of $n$ and $f$ improves simulation results. But with decreasing value of $t$ and increasing value of $n$ and $f$ the computational cost increases. Thus, the selection of the optimal parameters for DS simulations depends upon the trade-off between the CPU time and simulation quality.

\section{CONCLUSION AND RECOMMENDATION}

This study concludes that a novel S1 SAR dataset can be successfully used to retrieve the surface displacements of mountain glaciers employing a well-established DInSAR technique. Direct sampling, a newly developed MPS, is successful at deriving missing values in DInSAR derived displacement map of a glacier caused due to decorrelation of SAR images only using the information contained in nongapped area of the displacement map to be reconstructed. Both DS univariate and bivariate techniques provided acceptable results, well below the detection limit of DInSAR technique. With time, S1 database has grown with SAR images of a 6 day temporal baseline. In future works large number of displacement maps can be supplied as TIs offering better reconstruction results given the rich supply of spatial patterns from multiple TIs and investigate multivariate simulations to fill missing values in multiple DInSAR derived glacier displacement maps.

\section{REFERENCES}

Chen, C. W., \& Zebker, H. A. (2002). Phase unwrapping for large SAR interferograms: statistical segmentation and generalized network models. IEEE Transactions on Geoscience and Remote Sensing, 40(8), 1709-1719. http://doi.org/10.1109/TGRS.2002.802453

Joughin, I., Smith, B. E., \& Abdalati, W. (2010). Glaciological advances made with interferometric synthetic aperture radar. Journal of Glaciology, 56(200), 1026-1042. http://doi.org/10.3189/002214311796406158

Mariethoz, G., McCabe, M. F., \& Renard, P. (2012). Spatiotemporal reconstruction of gaps in multivariate fields using the direct sampling approach. Water Resources Research, 48(10), W10507. http://doi.org/10.1029/2012WR012115

Mariethoz, G., Renard, P., \& Straubhaar, J. (2010). The Direct Sampling method to perform multiple-point geostatistical simulations. Water Resources Research, 46(11), W11536. http://doi.org/10.1029/2008WR007621

Schneevoigt, N. J., Sund, M., Bogren, W., Kääb, A., \& Weydahl, D. J. (2012). Glacier displacement on Comfortlessbreen, Svalbard, using 2-pass differential SAR interferometry (DInSAR) with a digital elevation model. Polar Record, 48(1), 17-25. http://doi.org/10.1017/S0032247411000453

Yaseen, M., Hamm, N. A. S., Woldai, T., Tolpekin, V. A., \& Stein, A. (2013). Local interpolation of coseismic displacements measured by InSAR. International Journal of Applied Earth Observation and Geoinformation, 23(1), 1-17. http://doi.org/10.1016/j.jag.2012.12.002 